\title{
PRIMER REPORTE DE HEMISARCOPTES COOREMANI (THOMAS, 1961) (ACARI: HEMISARCOPTIDAE) PARA CUBA Y SU RELACIÓN CON CHILOCORUS CACTI L. (COLEOPTERA: COCCINELLIDAE)
}

\section{First report of Hemisarcoptes cooremani (Thomas, 1961) (Acari: Hemisarcoptidae) in Cuba and its relation with Chilocorus cacti L. (Coleoptera: Coccinellidae)}

\author{
Yadira Sánchez-Serrano ${ }^{1 a^{*}}$ y Abelardo Allán Méndez-Hernández ${ }^{1 \mathrm{~b}}$ \\ ${ }^{1}$ Centro Oriental de Ecosistemas y Biodiversidad (BIOECO), Cuba. a (i) orcid.org/0000-0002-1168-4693. \\ borcid.org/0000-0001-6184-7991, allan.biomail@gmail.com. *Para correspondencia: dira.acari@gmail.com
}

[Recibido: 16 de septiembre, 2021. Aceptado para publicación: 20 de octubre, de noviembre, 2021]

\section{RESUMEN}

La familia Hemisarcoptidae (Acari: Sarcoptiformes) se reporta por primera vez en Cuba, a partir de deutoninfas heteromórficas (hipopus) de Hemisarcoptes cooremani (Thomas, 1961) adheridas al cuerpo de pupas y una larva de Chilocorus cacti L. (Coleoptera: Coccinellidae), colectadas sobre cultivos de Carica papaya L. localizados en el municipio Segundo Frente, provincia Santiago de Cuba. Se ilustra y describe la morfología de los hipopus, así como su localización en el cuerpo de los primeros instares de C. cacti. Se abordan aspectos sobre la relación forética y mutualista entre ambas especies, detectada en este estudio desde estadios juveniles, un comportamiento con escasos registros. Esta asociación permitirá explorar nuevas estrategias para el control de plagas en Cuba, principalmente contra los insectos escama.

Palabras clave: ácaro, foresis, control de plagas, Diaspididae.

\section{ABSTRACT}

For the first time family Hemisarcoptidae and the non-native astigmatid mite Hemisarcoptes cooremani Thomas, 1961 is reported in the Cuban archipelago from heteromorphic deutonymphs (= hypopus), attached to pupae and one larva of Chilocorus cacti L. (Coleoptera: Coccinellidae), on Carica papaya L. crops located at Segundo Frente municipality, Santiago de Cuba province. The morphology of hypopus is well illustrated and described, as well as its location on the body of $C$. cacti first instars, a poorly approached behavior related with the phoretic and mutualist interactions between these two species, herein detected from early growth stages, a scarcely reported behavior. This mite-beetle association offers new strategies in order to apply better strategies for biological pest control programs in Cuba, mainly against scale insects.

Keywords: mite, phoresy, pest control, Diaspididae. 


\section{INTRODUCCIÓN}

Los ácaros de la familia Hemisarcoptidae Oudemans, 1904 pueden encontrarse asociados con una gran diversidad de insectos, ya sea como ectoparásitos depredadores de hemípteros plagas (Gerson et al., 2003), o en una relación forética o simbiótica con coleópteros, himenópteros, dípteros y colémbolos del género Sminthurus Latreille, 1802 (Fain et al., 1997).

Hasta el momento se han descrito 10 géneros dentro de Hemisarcoptidae: Congovidia Fain y Elsen, 1971; Divilia Sevastianov, 1969; Espeletiacarus Fain, 1987; Giardius Perraud, 1896; Hemisarcoptes Ligniéres, 1893; Huronopus OConnor y Houck, 1989a; Linobia Berlese, 1884; Nanacarus Oudemans, 1902; Pasohopus OConnor y Houck, 1989b y Superioropus OConnor y Houck, 1989a. Es una familia de distribución mundial, con mayor presencia de especies nativas en el hemisferio norte (p. ej. Estados Unidos, Canadá y Europa), así como en varias regiones de Asia, África, Australia (OConnor y Houck, 1989; Khaustov et al., 2018) y el norte de América del Sur, en Colombia (Fain, 1987). Únicamente Houck y OConnor (1996) refieren para "el Caribe y las Antillas" la presencia de Hemisarcoptes, aunque resulta un dato impreciso, sin localidad exacta, asumiendo al parecer la distribución más amplia de género y sus hospederos habituales (Chilocorus spp.). No obstante el género se ha reportado en la Florida (Smith y Dixon, 2008), y Hemisarcoptes malus se ha introducido como biocontrolador en las Islas Bermudas (Luck et al., 1999).

Varias especies de Hemisarcoptidae se han usado exitosamente para el control de plagas, tales como Linobia coccinellae Scopoli, 1763, ectoparásito de Chrysomela populi L. (Coleoptera: Chrysomelidae) (Pohlink et al., 2020) y las pertenecientes a Hemisarcoptes: H. coccophagus Meyer, 1962 H. malus (Shimer, 1868) y H. cooremani (Thomas, 1961), consideradas las más eficaces para el control de insectos escama (Hemiptera: Diaspididae), fundamentalmente de los géneros Aonidiella Berlese y Leonardi, 1895; Parlatoria Targioni-Tozzetti, 1869 y Lepidosaphes Shimer, 1898 (Erler y Tunq, 2001, Gerson et al., 2003, Dhooria, 2016), que constituyen reconocidas plagas de plantaciones de cítricos a escala mundial (Stimmel, 1996).

El género Hemisarcoptes es cosmopolita (Holte et al., 2001), en este solamente se dispersa la fase de deutoninfa heteromórfica o hipopus-propia del orden Sarcoptiformes, que presenta adaptaciones para adherirse a coccinélidos adultos, fundamentalmente del género Chilocorus Leach, 1815, con quienes mantiene una relación forética obligatoria hasta completar su desarrollo (Houck, 1999).

La especie Hemisarcoptes cooremani inicialmente se consideró como Vidia (Coleovidia) cooremani (Saproglyphidae) (Thomas, 1961), hasta su designación como sinonimia por Gerson (1967) y reubicada en Hemisarcoptidae. Varios estudios sugieren un vínculo exclusivo entre H. cooremani y Chilocorus cacti Linnaeus, 1767 (Houck y OConnor, 1990), sin embargo, esta especificidad género/especie-hospedero no es absoluta, reportándose asociaciones con los géneros Scymnus Kugelann, 1794; Halmus Mulsant, 1850 y Axion Mulsant, 1850 (Gerson et al., 1990). Gerson et al. (2003) además registró la colonización exitosa de H. cooremani de Texas, EE. UU. sobre adultos de Chilocorus nigritus (Fabricius) importados de la India, de donde son nativos.

En el caso de Chilocorus, sus especies son depredadoras de hemípteros como cóccidos, pseudocóccidosydiaspididos(Santiagoy Meneses-Lozano, 2010; Venegasetal.,2010),y deácaros como el Ácaro Rojo de la Palma Raoiella indica Hirst, 1924 (Trombidiformes: Tenuipalpidae) (Machkour-M'rabet et al., 2015). Chilocorus cacti ha mostrado ser la especie más eficaz 
para el control biológico de un gran número de plagas de hemípteros (p. ej. Aulacaspis yasumatsui, Lepidosaphes gloverii y Diaphorina citrii) y lepidópteros como Phyllocnistis citrella (Cave, 2006; Fernández et al., 2010). En Cuba se ha reportado en todas las provincias, depredando hemípteros tales como Pseudococcus spp. y Nipaecoccus nipae L. (Pseudococcidae), Coccus viridis Green, 1889 (Coccidae), Metaleurodicus spp. (Aleyrodidae) y Aphis spp. (Aphididae); así como lepidópteros del género Spodoptera, tisanópteros (Thrips palmi Karny, 1925 y Thrips tabaci Lindeman, 1888) y ácaros de la familia Tarsonemidae como Polyphagotarsonemus latus Banks, 1904 (Milán-Vargas et al., 2008).

La relación ecológica y evolutiva entre $H$. cooremani y $C$. cacti ha sido bien estudiada en países como Hungría (Fain y Ripka, 1998), España (Sorribas et al., 2008), China (Ji et al., 1994) y Estados Unidos (Miller et al., 2005); la misma ofrece numerosas ventajas, siendo aprovechado históricamente el uso simultáneo del ácaro y su hospedero para el control de plagas (Charles et al., 1995; Gerson y Izraylevich, 1997). El registro para Cuba de H. cooremani, asociado precisamente a C. cacti, incrementa las alternativas para el controlbiológico de los insectos escama, que ocasionan numerosas afectaciones agrícolas y grandes pérdidas financieras (González et al., 2005; Mestre-Novoa et al., 2015).

\section{OBJETIVOS}

- Presentar el primer reporte para Cuba de la familia Hemisarcoptidae y de Hemisarcoptes cooremani, abordando aspectos de su relación forética/mutualista con el coleóptero Chilocorus cacti.

\section{MATERIALES Y MÉTODOS}

Las deutoninfas de Hemisarcoptes cooremani (Fig. 1) se obtuvieron a partir de la colecta, en 2014, de una larva y diez pupas del coccinélido Chilocorus cacti (Fig. 2) en un cultivo de Carica papaya L. en el municipio Segundo Frente, provincia Santiago de Cuba, coordenadas 20²4'53,76”N - 75'32'18,76’W (Sistema de Coordenadas WGS84). Se contabilizaron todos los hipopus presentes en el frasco de colecta, tanto los dispersos en la solución alcohólica, como los adheridos aún a la superficie dorsal, ventral e interior de la larva (4to instar) y las pupas.

La larva y pupas de Chilocorus cacti se conservaron en alcohol al $70 \%$ y cinco ejemplares de $H$. cooremani se preservaron en preparaciones fijas en medio Hoyer según la metodología propuesta por Krantz (1978), para el análisis de caracteres morfológicos infragenéricos. Todos los especímenes referidos y preparaciones fijas se encuentran depositados en la colección zoológica del Departamento de Biología Animal del Centro Oriental de Ecosistemas y Biodiversidad (BIOECO), catalogados en los siguientes lotes: Hemisarcoptes cooremani (preservados en alcohol): BSCACRa.22,H. cooremani (preparaciones fijas):BSCACRp.201-205, Chilocorus cacti (larva y pupas): BSCE1.101.

Las deutoninfas de Hemisarcoptes cooremani se identificaron a partir de las descripciones originales de Thomas (1961), y de otras más detalladas de Krantz y Walter (2009) y Houck y OConnor (1990), de donde se revisaron algunas medidas de referencia de los caracteres diagnósticos (ver ejemplos en descripción de la deutoninfa). Se realizaron comparaciones 
morfológicas entre otras especies del género, usando los criterios y descripciones originales de Fain y Ripka (1998) para H. budensis, Meyer (1962) para H. coccophagus y Shimer (1868) para H. malus. Las categorías taxonómicas se actualizaron según Schatz (2011) y Walter et al. (2011). La identificación de C. cacti se realizó según Gordon y Hilburn (1990) y Rees et al. (1994).

Los especímenes de C. cacti se analizaron con un microscopio estereoscópico Carl Zeiss Stemi 2000-C equipado con una cámara digital Canon PowerShot A620 y para las preparaciones fijas de $H$. cooremani se utilizó un microscopio óptico Optech-B3 equipado con un sistema de captura de imágenes DCM-310. Para las mediciones, correspondientes únicamente al ejemplar fotografiado (Fig. 1), se usaron micrómetros oculares de escala lineal acoplados a cada dispositivo óptico, previamente calibrados con un micrómetro objetivo (Escala: $1 \mathrm{~mm}$ ). En el procesamiento de imágenes se utilizaron los programas CombineZP (actualización junio, 2010) para el ensamblaje de los diferentes planos de enfoque obtenidos y Adobe Photoshop C CS6 v13.0 para el revelado y composición fotográfica final.

\author{
RESULTADOS \\ Taxonomía \\ Phylum Arthropoda \\ Clase Arachnida \\ Subclase Acari \\ Superorden Acariformes Zakhvatkin, 1952 \\ Orden Sarcoptiformes Reuter, 1909 \\ Suborden Oribatida Van Der Hammen, 1968 \\ Infraorden Desmonomata Woolley, 1973 \\ Hipoorden Astigmata Canestrini, 1891 \\ Superfamilia Hemisarcoptoidea Oudemans, 1904 \\ Hemisarcoptidae Oudemans, 1904 \\ Hemisarcoptes Lignières, 1893 \\ Hemisarcoptes cooremani (Thomas, 1961)
}

\title{
Descripción y caracteres morfológicos
}

Todos los ejemplares de $H$. cooremani correspondieron a la fase de deutoninfa heteromórfica o hipopus (Fig. 1A, C y Fig. 2) y exhibían una coloración castaño oscuro. A partir del análisis de las preparaciones fijas se determinaron los siguientes caracteres diagnósticos: cuerpo ovoide fuertemente esclerotizado. Gnatosoma vestigial de forma triangular desarrollado, sin remanentes de quelíceros o palpos (Fig. 1A, C-gt), setas o solenidia cortas, cuatro pares de alveolos setales vestigiales (Fig. $1 \mathrm{C}-g t^{\prime}$ ).

Idiosoma fusiforme o redondeado con el contorno bien esclerotizado (longitud: $220 \mu \mathrm{m}[209.6 \mu \mathrm{m} \pm 11.7 \mu \mathrm{m} / 184 \mu \mathrm{m}-217.6 \mu \mathrm{m} \mathrm{n}=10$; en Houck y OConnor, 1990]), (ancho: $180 \mu \mathrm{m}[138 \mu \mathrm{m} \pm 11.0 / 124 \mu \mathrm{m}-156.8 \mu \mathrm{m} \mathrm{n}=10$; - Houck y OConnor, 1990]); placa sejugal presente. Esclerotización limitada de las placas coxales (Fig. 1A-C). Apodemas anteriores de las placas coxales I $\left(\mathrm{c}_{I}, a a_{I}\right)$ fusionado para formar un apodema esternal en forma de Y $\left(a e_{y}\right)$, que se extiende hasta las placas coxales II $\left(c_{I I}\right)$, y se conecta al apodema anterior II $\left(a a_{I I}\right)$ por 
una superficie esclerotizada. El apodema posterior I se encuentra fusionado al apodema anterior II $\left(a p_{I}, a a_{I I}\right)$. Apodema posterior II largo $\left(a p_{I I}\right)$, se extiende hasta el apodema anterior de la placa coxal III $\left(a a_{I I I}, c_{I I I}\right)$ sin fusionarse con él. Apodema anterior III medialmente convexo, conectado al apodema posterior medio $\left(a p_{m}\right)$ por una superficie esclerotizada. Apodema anterior IV $\left(a a_{I V}\right)$ no curvado, fusionado al apodema posterior medio, el cual se extiende caudalmente; el final posterior del apodema medio se bifurca y recurva lateralmente. Abertura genital ( $g e$ ) posterior a la placa coxal IV $\left(c_{I V}\right)$; seta genital filiforme $\left(s g e_{f}\right)$, flanqueando el extremo anterior de la abertura genital. Órgano oval de sujeción (os) flanqueado anteriormente por el apodema posterior de las placas coxales IV $\left(a p_{I V}\right)$. Ventosas anteriores y posteriores redondas $(v a)$. Ventosas cuticulares pareadas (anterior y lateral) $(v c)$ y ventosas cuticulares medias bien desarrolladas.

Patas anteriores I y II ( $p I$ y $p I I)$ relativamente largas $(98.8 \mu \mathrm{m}$ y $97.2 \mu \mathrm{m}$ respectivamente); patas III y IV reducidas ( $p I I I$ y $p I V)(67.3 \mu \mathrm{m}$ y $44.9 \mu \mathrm{m}$ respectivamente). Todos los segmentos son libres, excepto la tibia y el tarso de la pata IV que está fusionado (Fig. 1B -tf). El pretarso I-III (empodio - emp) consiste en una membrana ambulacral moderadamente larga con garras empodiales terminales $\left(g_{e m p}\right)(16.2 \mu \mathrm{m})$. En la pata IV el empodio y el pretarso no está presente. Seta $\left(s_{e}\right)$ ampliamente foliada, adherida a los tarsos de las patas I y II y unida con una seta $\left(s_{d}\right)$ filiforme corta; seta $\left(s_{s}\right)$ ausente en los tarsos I, II, and IV, en el tarso III está presente en forma de espina ventral gruesa. Seta coxal $\left(s_{w F}\right)$ adherida al fémur IV; las setas de la porción tarsal del tibio-tarso IV se conforman por una seta $\left(s_{r}\right)$ en forma de espina relativamente corta $\left(27.6 \mu \mathrm{m}\left[28.0 \mu \mathrm{m} \pm 3.2 \mu \mathrm{m} / 24.0 \mu \mathrm{m}-33.6 \mu \mathrm{m} \mathrm{n}=10 ;-\right.\right.$ Houck y OConnor, 1990]), una seta $\left(s_{d}\right)$ filiforme elongada en posición dorsal $(107.74 \mu \mathrm{m}[150.0 \mu \mathrm{m} \pm 11.2 \mu \mathrm{m} / 136.0 \mu \mathrm{m}-168.0 \mu \mathrm{m}$ $\mathrm{n}=10$; -Houck y OConnor, 1990]) y una seta $\left(s_{w}\right)$ filiforme más ancha y larga que $s_{d}$ (161.8 $\mu \mathrm{m}[172.9 \mu \mathrm{m} \pm 8.2 \mu \mathrm{m} / 168.0 \mu \mathrm{m}-184.0 \mu \mathrm{m} \mathrm{n}=10$; -Houck y OConnor, 1990]).

Se contabilizaron 55 deutoninfas dispersas en la solución alcohólica del frasco de colecta. En la larva de $C$. cacti se colectaron 25 hipopus, 15 de estos adheridos a los discos tergales y pliegues pleurales de la superficie externa (Fig. 2A), así como otras 10 en el interior del abdomen, al cual se accedió a partir de una incisión pre existente en la región dorsal del pronoto.

Entre todas las pupas de C. cacti analizadas se contabilizaron 735 hipopus de H. cooremani, colectándose en una de ellas un total de 220 , la mayor cantidad adheridas a la superficie ventral de la cabeza, el proesterno, el mesoesterno y algunos segmentos abdominales (Fig. 2C, D y E); estas regiones, en las pupas de Chilocorus (y la mayoría de coccinélidos) permanecen parcialmente protegidas dentro de la exuvia larval hasta su metamorfosis en adulto (Ponsonby y Copland, 1997), (Fig. 2B). 

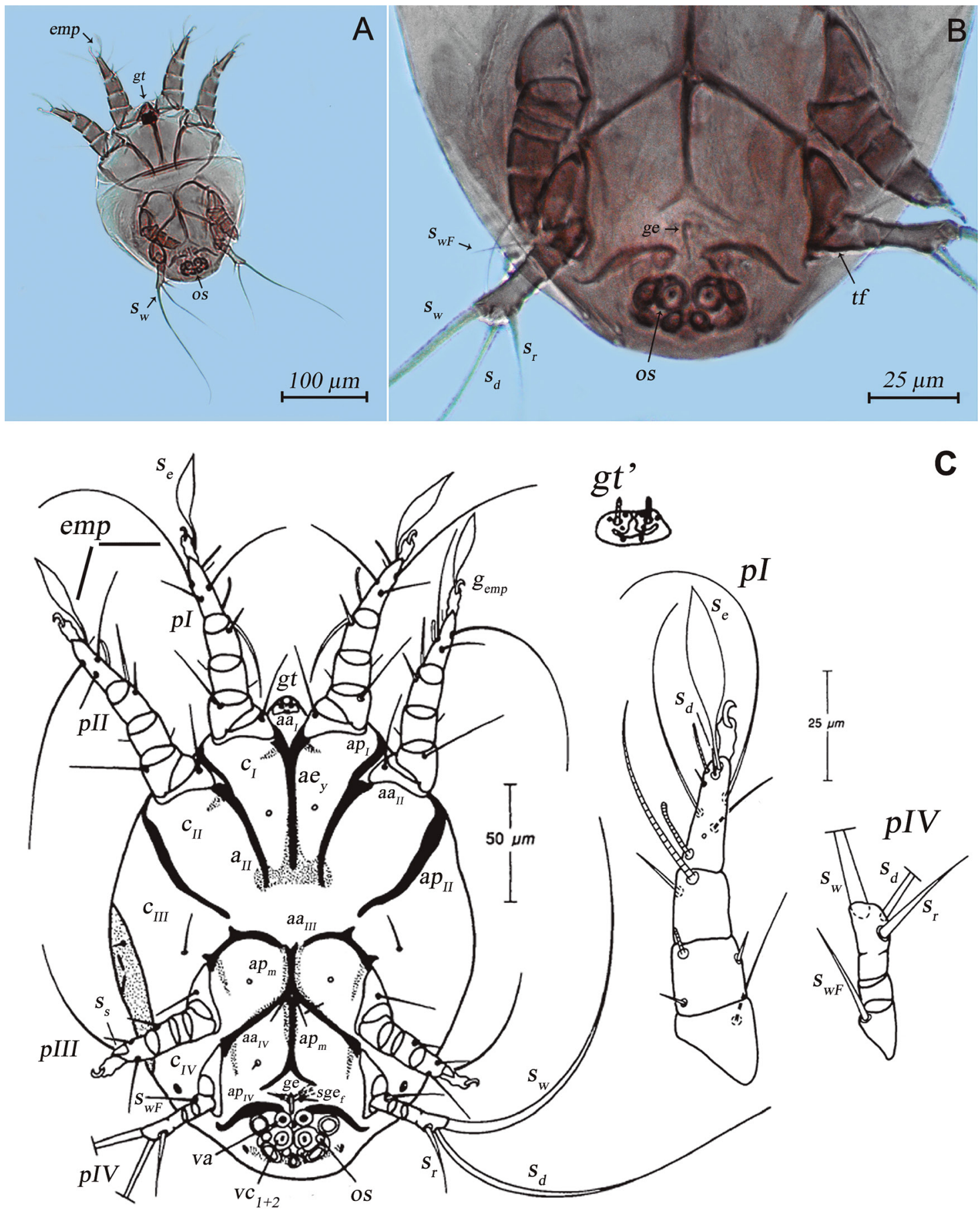

Figura 1. Deutoninfa heteromórfica de Hemisarcoptes cooremani, características diagnósticas. A, Vista ventral; B, Ampliación de la región ventral. C, Dibujo esquemático con detalle de la vista ventral del idiosoma y de las patas I y IV (Modificado de Houck y OConnor, 1990). Abreviaturas: gt: gnatosoma vestigial, cI-IV: placa coxal, aaI-IV: apodema anterior, apI-II, IV: apodema posterior, aey: apodema esternal en forma de Y, ge: abertura genital, sgef: seta genital filiforme, os: órgano oval de sujeción, va: ventosas anteriores, vc: ventosas cuticulares. $\mathrm{pI}-\mathrm{IV}$ : patas anteriores y posteriores, tf: segmento tarsal fusionado, emp: empodio, gemp: garra empodial, se: seta empodial foliada, sd: seta empodial filiforme corta, swF: seta coxal, sr: seta tarsal en forma de espina, sd: seta tarsal filiforme, sw: seta tarsal filiforme elongada. 

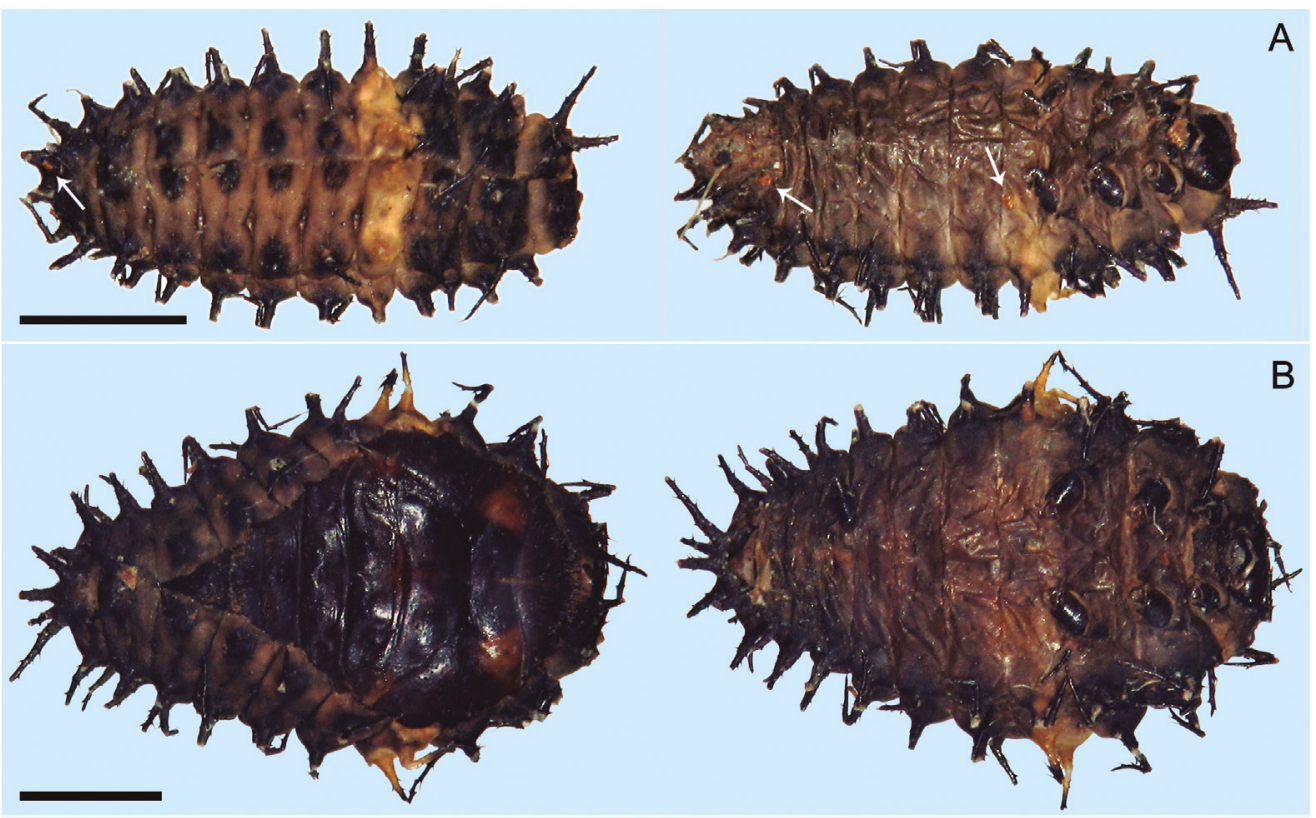

B
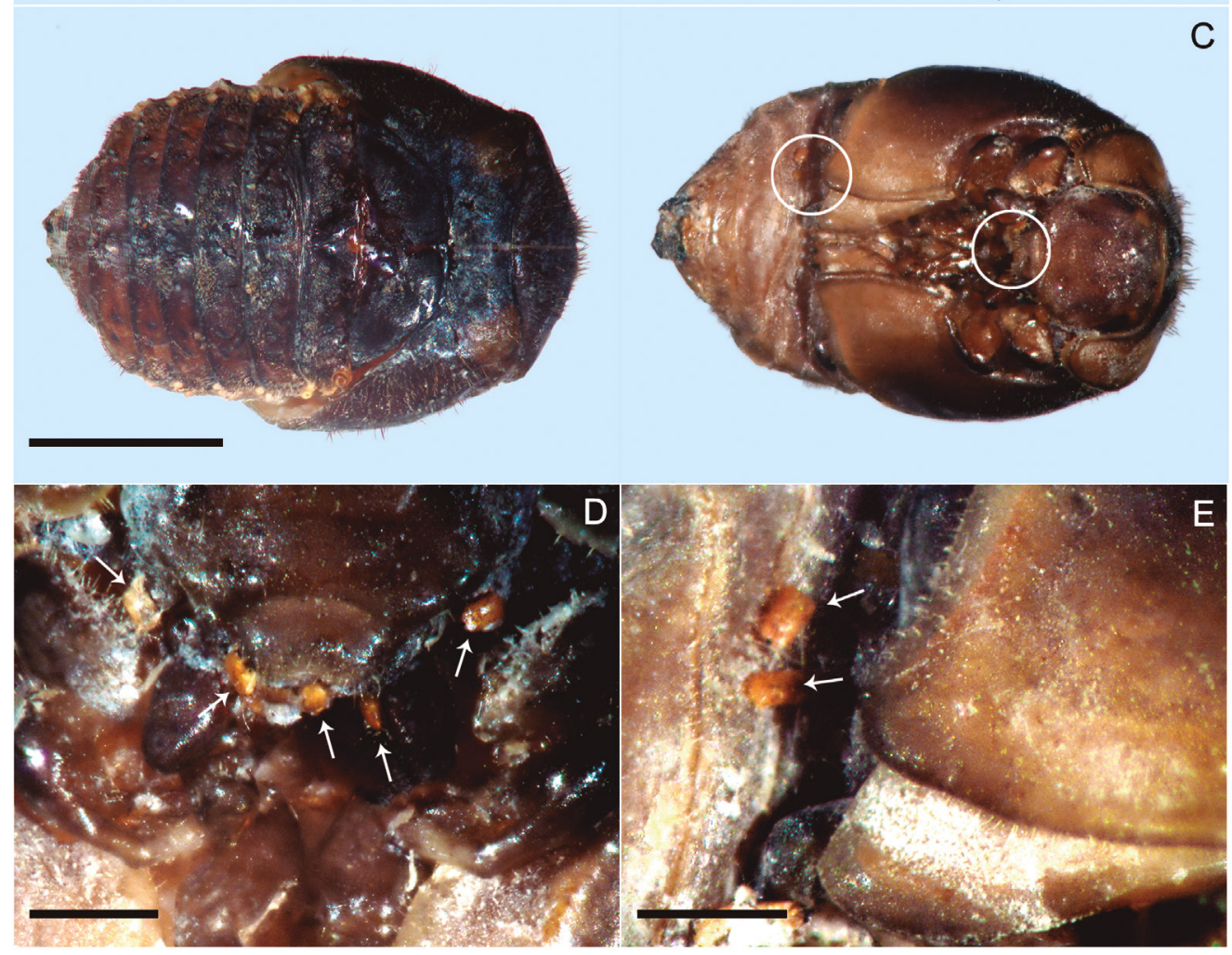

Figura 2. Vistas dorsal y ventral de la larva y pupas de Chilocorus cacti. A, larva; B, C: pupa en el interior de la exuvia (B) y extraída de la misma: (C). Escala: $2 \mathrm{~mm}$. D-E, Superficie ventral de la cabeza, el proesterno y mesoesterno (D) y los segmentos abdominales (E). Escala: 0,5 mm. Los círculos y flechas indican la localización exacta de los hipopus de Hemisarcoptes cooremani. 


\section{DISCUSIÓN}

Este constituye el primer registro de la familia Hemisarcoptidae para Cuba, representada por Hemisarcoptes cooremani, en asociación con el coccinélido Chilocorus cacti. Anteriormente, H. cooremani se había reportado desde el sur de Estados Unidos hasta el norte de América del Sur, incluyendo el Caribe de forma imprecisa (Houck y OConnor, 1996), basándose probablemente en el rango de distribución estimado de la especie y de su hospedero habitual (Houck y OConnor, 1990). No obstante, no se descarta alguna introducción antrópica no reportada con motivos de control biológico en alguna isla del Caribe, actividad que a día de hoy no ha sucedido en Cuba.

En este trabajo se contabilizaron un máximo de 220 deutoninfas en una pupa de Chilocorus cacti, valores similares a los encontrados por otros autores, aunque en individuos adultos de especies del mismo género. Gerson y Schneider (1982) reportan un promedio de 30 individuos (máximo: 202) de H. coccophagus sobre un ejemplar de C. bipustulatus (L.). Houck (1994) registró un promedio de 300 hipopus (máximo: 800) de $H$. cooremani sobre individuos de C. cacti y Gerson (1994) para C. flavidus Blackburn y C. australasiae Kerville promedios de 250 (máximo: 615) y 217 (máximo: 847) respectivamente de Hemisarcoptes sp. Estas deutoninfas no causan ningún efecto perjudicial sobre sus hospederos, aunque en altas densidades pueden ralentizar sus movimientos (Gerson, 1967).

Inicialmente reconocida por Bartlett y DeBach (1952) en California, la presencia de deutoninfas se ha reportado casi exclusivamente en coccinélidos adultos; en nuestro estudio se localizaron en la superficie corporal e interior de una larva y pupas, un hecho inusual del cual no se encontraron registros en la literatura. Este hallazgo confirma la migración activa de las deutoninfas durante su ciclo de vida y en dependencia del estadio del coleóptero, esto es, en una primera instancia hacia la protección que ofrece el espacio entre la exuvia y la pupa, y posteriormente hacia la superficie interna de los élitros ya desarrollados, donde se concentran fundamentalmente a lo largo del margen epipleural, evitando las superficies con alta densidad de microsetas y espinas que puedan dañar la placa de sujeción (Fig. 1A y C $-O s$ ) (Izraylevich y Gerson, 1995; Houck, 1994, 1998, 1999).

Si bien se reconoce ampliamente la relación forética entre ambas especies, varios autores la clasifican como parasítica o mutualista (Houck y OConnor, 1991; Izraylevich et al., 1995), donde $H$. cooremani por medio de la abrasión causada por las ventosas caudoventrales (Fig. 1B 'ven') en la hipodermis, genera un canal que permite el flujo bidireccional tanto de sustancias propias del coleóptero (p. ej. hemolinfa) como de enzimas, hormonas o azúcares excretados por el ácaro (Holte et al., 2001). Según Houck y Cohen (1995) constituye esta una estrategia tipo 'lobo en piel de cordero' (wolf-in-sheep's-clothing'), que le ofrece a los ácaros varias ventajas: la adquisición efectiva de nutrientes les permite un desarrollo más rápido, pudiendo mudar del primer al tercer instar ninfal (protoninfa a tritoninfa), sin pasar por la etapa de deutoninfa heteromórfica (Houck y OConnor, 1991); facilita su dispersión hacia áreas distantes y el acceso a nuevas fuentes de alimentos, así como aprovechar la composición alcaloidea tóxica de la hemolinfa del coleóptero, inocua para los hipopus, como un mecanismo de defensa efectivo (McCormick et al., 1994; Xiongwei et al., 1995). 


\section{CONCLUSIONES}

La detección por primera vez en Cuba del ácaro Hemisarcoptes cooremani, asociado a los primeros estadios de su hospedero habitual Chilocorus cacti, reafirma la interdependencia (forética/mutualista) existente entre ambas especies, pudiendo su uso conjunto incrementar el éxito en el control de plagas como los hemípteros diaspididos, de los que son potenciales depredadores.

\section{AGRADECIMIENTOS}

A los acarólogos Pedro de la Torre Santana y Lérida Almaguel Rojas. A Liet Torres Osorio (Director de la Estación Fitosanitaria de Segundo Frente, Santiago de Cuba) y Ada Iris Gonzales Reyes por facilitar la muestra. A Adrián González-Guillén y otros revisores anónimos por sus acertadas recomendaciones.

\section{LITERATURA CITADA}

Bartlett, B. y P. DeBach. 1952. New natural enemies of avocado pests. Citrus Leaves, 32 (10): 16-7.

Berlese, A. 1884. La sistematica dei Sarcoptidi. Bolletino della Società Entomologica Italiana, 16: $287-292$.

Cave, R. D. 2006. Biological control agents of the cycad aulacaspis scale, Aulacaspis yasumatsui. Proceedings of the Florida State Horticultural Society, 119: 422-424.

Charles, J. G., M. G. Hi y D. J. Allan. 1995. Persistence of the predatory mite, Hemisarcoptes coccophagus Meyer (Hemisarcoptidae), on low populations of Hemiberlesia lataniae (Signoret) (Diaspididae) in New Zealand. Israel Journal of Entomology, 29: 297-300.

Dhooria, M. S. 2016. Mites Predaceous on Pests of Agriculture. En: Fundamentals of Applied Acarology, pp. 363-379. Singapore, Springer Singapore.

Erler, E. y I. Tunq. 2001. A Survey (1992-1996) of natural enemies of diaspididae species in Antalya, Turkey. Phytoparasitica, 29 (4): 299-305.

Fain, A. 1987. Notes on the mites living in the flowers of Espeletia spp. (Asteraceae) in Colombia. II. Espeletiacarus andinus gen. n., sp. n. (Hemisarcoptidae) and Michaelopus incanus sp. n. (Acaridae). Entomologische Mitteilungen Zoologische Museum Hamburg, 9 (130): 37-48.

Fain, A. y G. Ripka. 1998. A new species of Hemisarcoptes Ligniéres, 1893 (Acari: Hemisarcoptidae) from ornamental trees in Hungary. International Journal of Acarology, 24 (1): 33-39.

Fain, A. y P. Elsen. 1971. Notes sur les hypopes des Saproglyphidae (Acarina: Sarcoptiformes). Revue de Zoologie et de Botanique africaines, 94: 281-284.

Fain, A., G. D. Hurst, C. Fassotte, K. M. Webberley, J. J. Sloggett y M. E. Majerus. 1997. Entomologie Bulletin del L'Institut Royal des Sciences Naturelles de Belgique, 67: 89-94. 
Fernández, C. G., M. G. Pacheco, D. H. Espinosa y J. R. Tapia. 2010. Entomófagos asociados a las plagas citrícolas, Lepidosaphes gloverii Packard (Hemiptera: Diaspididae), Phyllocnistis citrella Stainton (Lepidoptera: Gracillariidae) y Diaphorina citrii (Kuwayama) (Hemiptera: Psyllidae) en naranjo Valencia. Centro Agrícola, 37 (4): 75-81.

Gerson, U. 1967. Observations on Hemisarcoptes coccophagus Meyer (Astigmata: Hemisarcoptidae), with a new synonym. Acarologia, 9: 632-638.

Gerson, U. 1994. First record of the genus Hemisarcoptes Lignières (Acari: Astigmata: Hemisarcoptidae) in Australia. Australian Entomology, 21: 71-4.

Gerson, U. y R. Schneider. 1982. The hypopus of Hemisarcoptes coccophagus Meyer (Acari: Astigamata: Hemisarcoptidae). Acarologia, 23 (2): 171-176.

Gerson, U. y S. Izraylevich, 1997. A review of host utilization by Hemisarcoptes (Acari: Hemisarcoptidae) parasitic on scale insects. Systematic and Applied Acarology, 2: 33-42.

Gerson, U., B. M. OConnor y M. A. Houck. 1990. Acari. En: World Crop Pests, Volume $4 B$. Armoured Scale Insects: Their Biology, Natural Enemies and Control (Rosen, D. Ed.). Elsevier, Amsterdam, The Netherlands.

Gerson, U., R. L. Smiley y O. Ronald. 2003. Mites (Acari) for Pest Control. Blackwell Science Ltd. Berlin, Germany.

González, C., S. Cáceres, M. Gómez, M. Fernández, D. Hernández y J. Tapia. 2005. Lepidosaphes gloverii (Hemiptera: Diaspididae): estudios biológicos y ecológicos en cítricos de Cuba. Revista de la Sociedad Entomológica Argentina, 64 (1/2): 26-28.

Gordon, R. D. y D. J. Hilburn. 1990. The Coccinellidae (Coleoptera) of Bermuda. Journal of New York Entomology Society, 98 (3): 265-309.

Holte, A., M. Houck y N. Collie. 2001. Potential role of parasitism in the evolution of mutualism in astigmatid mites: Hemisarcoptes cooremani as a model. Experimental \& Applied Acarology, 25: 97-107.

Houck, M. A. 1994. Adaptation and transition into parasitism from commensalism: a phoretic model. En: Mites: Ecological and Evolutionary Analyses of Life History Patterns (Houck, M. A. Ed.), pp. 252-281. Chapman \& Hall, New York.

Houck, M. A. 1998. Subelytral ultrastructure of Chilocorus (Coleoptera: Coccinellidae): Influence on phoresy by Hemisarcoptes (Acari: Hemisarcoptidae). Experimental \& Applied Acarology. 22: 1-22.

Houck, M. A. 1999. Review: phoresy by Hemisarcoptes (Acari: Hemisarcoptidae) on Chilocorus (Coleoptera: Coccinellidae): influence of subelytral ultrastructure. Experimental \& Applied Acarology, 23 (1999): 97-118.

Houck, M. A. y A. C. Cohen. 1995. The potential role of phoresy in the evolution of parasitism: radiolabeling (tritium) evidence from an astigmatid mite. Experimental \& Applied Acarology, 19: 677-694. 
Houck, M. A. y B. M. OConnor. 1990. Ontogeny and life history of Hemisarcoptes cooremani (Acari: Hemisarcoptidae). Annals of the Entomological Society of America, 83 (5): 869-886.

Houck, M. A. y B. M. OConnor. 1991. Phoresy in the acariform Acari. Annual Revisions in Entomology, 36: 611-636.

Houck, M. A. y B. M. OConnor. 1996. Temperature and host effects on key morphological characters of Hemisarcoptes cooremani and Hemisarcoptes malus (Acari: Hemisarcoptidae). Experimental \& Applied Acarology, 20 (1996): 667-682.

Izraylevich, S. y U. Gerson. 1995. Spatial patterns of the parasitic mite Hemisarcoptes coccophagus (Astigmata: Hemisarcoptoidae): host effect, density-dependence of aggregation, and implications for biological control. Bulletin of Entomological Research, 85: $235-240$.

Izraylevich, S., O. Hasson y U. Gerson. 1995. Frequency-dependent host selection by parasitic mites: a model and a case study. Oecologia, 102: 138-145.

Ji, L., U. Gerson y S. Izraylevich. 1994. The mite Hemisarcoptes sp. (Astigmata: Hemisarcoptidae) parasitizing willow oyster scale (Homoptera: Disapididae) on poplars in Northern China. Experimental \& Applied Acarology, 18: 623-627.

Khaustov, A. A., P. B. Klimov, V. A. Trach, A. N. Bobylev, V. M. Salavatulin, V. A. Khaustov y A. V. Tolstikov. 2018. Review of mites (Acari) associated with the european spruce bark beetle, Ips typographus (Coleoptera: Curculionidae: Scolytinae) in Asian Russia. Acarina, 26 (1): $3-79$.

Krantz, G. 1978. A Manual of Acarology 2nd Ed. Oregon State University Book Stores, Corvallis.

Krantz G. y E. Walter. 2009. A Manual of Acarology 3rd Ed. Texas Tech University Press, Lubbock.

Lignières, J. 1893. Etude zoologique et anatomique de l'Hemisarcoptes. Mémories de la Societé Zoologique de France, 6: 16-25.

Luck, R. F., G. Jiang y M. A. Houck, 1999. A laboratory evaluation of the astigmatid mite Hemisarcoptes cooremani Thomas (Acari: Hemisarcoptidae) as a potential biological control agent for an armored scale, Aonidiella aurantii (Maskell) (Homoptera: Diaspididae). Biological Control, 15: 173-183.

Machkour-M'rabet, S., J. Ferral-Piña y Y. Henaut. 2015. Chilocorus cacti (Coleoptera: Coccinellidae), enemigo natural potencial del ácaro rojo de las palmas en México. Acta Zoológica Mexicana, 31 (3): 512-517.

McCormick, K. D., A. B. Attygalle, S. C. Xu, A. Svatos, J. Meinwald, M. A. Houck, C. L. Blankespoor y T. Eisner. 1994. Chilocorine: heptacyclic alkaloid from a coccinellid beetle. Tetrahedron, 50: 2365-2372.

Mestre-Novoa, N., A. Hamon, G. Hodges y T. Kondo. 2015. Lista de los insectos escama (Hemiptera: Sternorrhyncha: Coccoidea) de Cuba. Poeyana, 500 (enero-junio): 33-54. 
Meyer, K. P. 1962.Two new mite predators of red scale (Aonidiella aurantii) in South Africa. South African Journal of Agricultural Science, 5 (3): 411-417.

Milán-Vargas, O., N. Cueto-Zaldívar, N. Hernández-Pérez, T. Ramos-Torres, M. PinedaDuvergel, R. Granda-Sánchez, M. Peñas-Rodríguez, J. Díaz del Pino, S. Caballero-Figueroa, I. Esson-Campbell, T. Corona-Santos, L. A. Rodríguez-Ramírez, J. L. de Armas-García, J. M. Montalvo-Guerrero y E. Delís-Hechavarría. 2008. Prospección de los coccinélidos benéficos asociados a plagas y cultivos en Cuba. Fitosanidad, 12 (2): 71-78.

Miller, D. R., G. L. Miller, G. S. Hodges y J. A. Davidson. 2005. Introduced scale insects (Hemiptera: Coccoidea) of the United States and their impact on U.S. agriculture. Proceedings of Entomological Society of Washington, 107 (1): 123-158.

OConnor, B. M. y M. A. Houck. 1989a. Two new genera of Hemisarcoptidae (Acari: Astigmata) from the Huron Mountains of Northern Michigan. Great Lakes Entomology, 22: 1-10.

OConnor, B. M. y M. A. Houck. 1989b. A new genus and species of Hemisarcoptidae from Malaysia (Acari: Astigmata). International Journal of Acarology, 15: 17-20.

Oudemans, A. C. 1902. Acarologische Aanteekeningen. Entomologische Berichten I, 7: 43-45.

Perraud, J. 1896. An acarine parasite of the vine. Comptes Rendus des Seances de la Societe de Biologie et de Ses Filiales, 3: 10.

Pohlink, K., R. Georgi y M. Müller. 2020. Occurrence and impact of the parasitic mite Linobia coccinellae on its host beetle Chrysomela populi: implications for its potential as a biological control agent. Entomologia Experimentalis et Applicata, 168: 723-731.

Ponsonby, D. J. y J. W. Copland. 1997. 2.2 - Chapter 2.2 Predators: 2.2.1 Coccinellidae and other Coleoptera. En: World Crop Pests: Soft Scale Insects their Biology, Natural Enemies and Control Volume 7, Part B, pp. 3-442 (Ben-Dov, Y. y C. J. Hodgson Ed.), pp. 29-60. Elsevier.

Rees, B. E, D. M. Anderson, D. Bouk y R. D. Gordon. 1994. Larval key to genera and selected species of North American Coccinellidae (Coleoptera). Proceedings of the Entomological Society of Washington, 96 (3): 387-412.

Santiago, E. C. y H. M. Meneses-Lozano. 2010. Red gold-raising cochineal in Oaxaca. Textile Society of America Symposium Proceedings, Paper 39.

Schatz, H. 2011. Suborder Oribatida van der Hammen, 1968. En: Animal Biodiversity: An Outline of Figher-level Classification and Survey of Taxonomic Richness. Zootaxa Special Issue (Zhang, Z. Q. Ed.), pp. 141-148.

Sevastianov, V. D. 1969. A new myrmecophilus mite Divilia oculata gen. et sp. nov (Sarcoptiformes: Saproglyphidae). Zoologische Journal, 48: 447-449 (in Russian).

Shimer, H. 1868. Notes on the 'Apple bark-louse' (Lepidosaphes conchiformis, Gmelin sp.) with a description of a supposed new Acarus. Transactions of the American Entomological Society, 1: 361-374. 
Smith, T. R. y W. N. Dixon. 2008. 2007 Florida CAPS Red Palm Mite Survey 2nd Interim Report. October 2006 to January 2008. Florida Cooperative Agriculture Pest Survey. Program Report No. 2007-02-RPM-02.

Sorribas, J. J., R. Rodríguez, E. Rodrigo y F. García-Marí. 2008. Parasitism levels and species of natural enemies in field populations of California red scale Aonidiella aurantii (Hemiptera: Diaspididae) in eastern Spain. Integrated Control in Citrus Fruit Crops Bulletin, 38: 26-33.

Stimmel, J. 1996. A large scale dilemma. American Nurseryman, March: 38-45.

Thomas, H. A. 1961. Vidia (Coleovidia) coaremani, new subgenus and new species, and notes on the life history (Acarina: Saproglyphidae). Annals of Entomological Society of America, 54: 461-463.

Venegas, R. J. M., J. R. Lomelí-Flores, E. Rodríguez-Leyva, G. Mora-Aguilera y J. M. Valdéz. 2010. Enemigos naturales de Dactylopius opuntiae (Cockerell) en Opuntia ficus-indica (L.) en el centro de México. Acta Zoológica Mexicana, 26 (2): 415-433.

Walter, D. E., S. Bolton, M. Uusitalo y Z. Q. Zhang. 2011. Suborder Endeostigmata Reuter, 1909. En: Animal biodiversity: An Outline of Higher-level Classification and Survey of Taxonomic Richness (Zhang, Z. Q. Ed.), Zootaxa Special Issue, pp. 139-140.

Xiongwei, S., A. B. Attygalle, A. J. Meinwald, M. A. Houck y T. Eisner. 1995. Spirocyclic defensive alkaloid from a coccinellid beetle. Tetrahedron, 51: 8711-8718.

Cómo citar: Sánchez-Serrano, Y., \& Méndez-Hernández, A. A. (2022). Primer reporte de Hemisarcoptes cooremani (Thomas, 1961) (Acari: Hemisarcoptidae) para Cuba y su relación con Chilocorus cacti L. (Coleoptera: Coccinellidae). Novitates Caribaea, (19), 92-104. https://doi.org/10.33800/nc.vi19.280. Artículo científico original. 